\title{
Protective role of Nigella sativa against experimentally induced type-I I diabetic nuclear damage in Wistar rats
}

\author{
T. J. Sheikh, D. V. Joshi, B. J. Patel and C. M. Modi \\ Department of Veterinary Pathology, \\ College of Veterinary Science and A.H, S. D. Agricultural University, Sardarkrushinagar - 385506, Gujarat, India \\ Corresponding author: T. J. Sheikh, email: drsheikh2@yahoo.co.in \\ Received: 10-04-2013, Revised: 24-04-2013, Accepted: 24-04-2013, Published online: 28-07-2013
}

doi: $10.14202 /$ vetworld.2013.698-702

How to cite this article: Sheikh TJ, J oshi DV and Patel BJ (2013) Protective role of Nigella sativa against experimentally induced type-II diabetic nuclear damage in Wistar rats, Veterinary World 6(9): 698-702.

\begin{abstract}
Aim: To identify the anti-mutagenic effect of Nigella sativa on the experimentally induced chronic diabetes (type - II) in Wistar rats.

Materials and Methods: The anti-mutagenic effect was evaluated in Nigella sativa treated diabetic rats against the streptozotocin - nicotinamide (STZ-NA) (at a dose rate of 45-110 i.p mg/kg b.wt for 90 days) induced type-II diabetes mellitus using bone marrow micronucleus tests. The antioxidant status was tested by estimating the serum levels of lipid peroxidation and superoxide dismutase.

Results: Our results indicated that diabetic rats treated with Nigella sativa decreased the frequency of micronuclei in the erythrocytes of bone marrow $(P<0.05)$ and enhanced the antioxidant status $(P<0.05)$ in the treated diabetic rats as compared to controls.

Conclusion: The observations indicated that the diabetic patients are more prone to cell mutations which are related to the level of cellular oxidative status and it could be reduced by Nigella sativa.
\end{abstract}

Keywords: antioxidant, diabetes, micronucleus test, Nigella sativa, plant extract

\section{I ntroduction}

Diabetes mellitus is a metabolic disorder and is recognized as one of the leading causes of death and disability worldwide [1]. It results from a reduction of insulin levels available for normal function of many cells in the body which subsequently impairs or retards the cellular metabolism of carbohydrates, proteins and lipids -an effect which results in long term health complications [2-3]. Type 2 diabetes mellitus (T2DM) affects about $12 \%$ of the Indian population [4]. Higher prevalence, morbidity and mortality through T2DM occur throughout the world and thus, this disease warrants investigations for designing effective prevention and control measures.

Evidence from both clinical and experimental studies indicates that there is a close link between hyperglycemia, oxidative stress related damage and diabetic complications [5]. Hyperglycemia plays a role in the overproduction of reactive oxygen species by the mitochondrial electron transport chain. Reactive oxygen species virtually damage all cellular components, leading to DNA and protein modifications and lipid peroxidation [5]. In addition, diabetic patients are reported to have a reduced superoxide dismutase activity. As a consequence, they not only suffer from an oxidative stress related diseases but are also likely to transmit the nuclear defects to their subsequent progeny [6].

This article is an open access article licensed under the terms of the Creative Commons Attribution License (http://creativecommons. org/licenses/by/2.0) which permits unrestricted use, distribution and reproduction in any medium, provided the work is properly cited.
Administration of nicotinamide (NA)-streptozotocin (STZ) induced moderate hyperglycemia which has clinical similarities especially with respect to the insulin response to the glucose. The partial protection of beta-cells by the nicotinamide against the cytotoxic action of the streptozotocin is reported to play a major role in the development of non-insulin dependent diabetes mellitus (NIDDM) condition in rats [7].

Genetic instability, impaired DNA repairing mechanisms and subsequent risks to health such as inheritable disease, neoplasm are observed in diabetes mellitus patients [6]. Studies based on mutations assume importance because the DNA alterations can lead to several inheritable diseases [9]. Reduced antioxidant defense in T2DM could be an important factor underlying this association. Micronuclei are small nuclei that develop from chromosomal fragments or from intact whole chromosomes that lag behind during the anaphase stage of cell division. Micronuclei (cytoplasm chromatin masses) with the appearance of small nuclei in cells are a reflection of structural and/or numerical chromosomal aberrations arising during mitosis and are regarded as promising biomarkers to assess the genotoxicity or impaired DNA repair [10]. Among the battery of tests available, in vivo bone marrow micronucleus test is the most commonly employed test to evaluate the drug/disease induced mutations [11]. Plant extracts, generally Indian spice is used as an anti-diabetic agent in the therapy of noninsulin dependent diabetes mellitus (NIDDM).

However, anti-diabetic drugs likes, pioglitazone and glimepiride combination does not protect the cells 
from nuclear damage, i.e. micronuclei formation and sperm abnormalities in NA-STZ induced diabetic rats [12], indicating the need to assess the contribution of anti-diabetic plant extract drugs towards reducing genotoxicity and cellular damages. Besides, there has been an increasing use of natural antioxidants from plant extracts as a means to prevent oxidative damage in diabetic patients due to high oxidative stress. Naturally occurring phytochemicals with anti-diabetic activities are relatively nontoxic, inexpensive and are available in an ingestive form. Therefore, they are commonly used to prevent morbidity and mortality from chronic diseases in countries where low or middle-income populations are high in numbers [13]. The seed of Nigella sativa, an annual herbaceous plant, has been used for centuries as a traditional treatment for asthma. Clinical and experimental studies have demonstrated many useful therapeutic effects of Nigella sativa extracts, including anti-diabetic effect [14].

As per our knowledge, no published reports on protective effect of Nigella sativa in STZ-NA induced genotoxicity are available, indicating the need to assess the protective effect of Nigella sativa extract as an antidiabetic drug towards genotoxicity and cellular damages.

\section{Materials and Methods}

Chemicals: The streptozotocin- nicotinamide, other reagents/chemicals and stains utilized in this study were of analytical grade and were procured from Himedia Labs (P) Ltd, Mumbai.

Animals: Ten to twelve week-old healthy, laboratory in-bred, male Wistar rats weighing $230 \pm 20 \mathrm{~g}$ were maintained under standard laboratory conditions such as temperature $20 \pm 2{ }^{\circ} \mathrm{C}, 12 \mathrm{~h}$ light / dark cycle and were provided with water and pellet feed (Amrut rat and mice feed, Pranav Agro Industries Ltd., Sangli, India) ad libitum .

Ethical approval: The experimental trial was conducted after obtaining the formal approval from the regional animal ethics panel.

Induction of type-II diabetes: Experimental type-II diabetes mellitus (T2DM) was induced in adult rats by administering nicotinamide (NA) and streptozotocin (STZ) [7]. The animals received intraperitoneal administration of Nicotimamide @ $110 \mathrm{mg} / \mathrm{kg}$ dissolved in saline $15 \mathrm{~min}$ before the administration of STZ $-45 \mathrm{mg} / \mathrm{kg}$, i.p dissolved in $0.1 \mathrm{M}$ citrated buffer (pH 4.5) immediately before use. Blood glucose levels were estimated by glucometer after 3 days of STZ-NA induction and the animals with glucose level > 180 $\mathrm{mg} / \mathrm{dl}$ were only selected for the study. A moderate hyperglycemia was found in rats treated with NA and STZ.

Nigella sativa (Black cumin) seed aqueous extract: Seeds of Nigella sativa were washed multiple times with clean water then the seeds were dried under shade for one week at $25^{\circ} \mathrm{C}$ with frequent turning over. The seeds were then pulverized in a grinder to obtain fine powder. The aqueous seeds extract was prepared by mixing $100 \mathrm{gm}$ of the powder with $200 \mathrm{ml}$ of distilled water using a magnetic stirrer. The mixture was then filtered and lyophilized. Stock solution was prepared by dissolving $600 \mathrm{mg}$ of lyophilized powder in $10 \mathrm{ml}$ of distilled water. From this stock solution, the required concentrations of the extract were prepared for the study [15].

Experimental design: The experiment was conducted for 90 days. The animals were divided into following three groups. Each group contained 10 animals.

Group I (Normal Control animals) - treated with vehicle alone (normal saline) i.p, once

Group II (Diabetic animals) - STZ-NA @ 45-110 $\mathrm{mg} / \mathrm{kg}$ i.p, once

Group III (diabetic- Nigella sativa) - 500 mg/kg P.O daily

Bone marrow micronucleus test: At the end of experiment the femur was removed from each rat, the ends were clipped to expose the bone marrow and smears were prepared. The femoral cells were flushed out with fetal bovine serum containing $25 \mathrm{mmol} / \mathrm{litre}$ EDTA, filtered through a cloth and centrifuged at $200 \times$ $\mathrm{g}$ for $5 \mathrm{~min}$. The supernatant was removed, the cell pellet was carefully mixed by repeated aspiration with a Pasteur pipette and a small drop was spread onto a clean microscope slide, air-dried and then fixed with methanol. After drying again, slides were dipped in fresh phosphate buffer, pH 6.4, stained with geimsa and examined for the presence of micronuclei. The micronuclei (MN) were identified in two forms of RBCs (i.e. polychromatic erythrocytes (PCEs) and normochromatic erythrocytes (NCEs)). About 2000 PCEs and corresponding NCEs were scrutinized for the presence of micronuclei. The PCEs divided with corresponding NCEs per animal was used to calculate the $\mathrm{P} / \mathrm{N}$ ratio [16].

In vivo antioxidant activity: Blood samples were collected from the retro-orbital plexus under ether anesthesia. The packed cells were separated by centrifugation and immediately analyzed to determine the antioxidant enzyme activity.

Lipid peroxidation (LPO): Membrane peroxidative damage in erythrocytes was determined in terms of malondialdehyde (MDA) production as per the method described by Shafiq-U-Rehman [17]. The underlying principle is the reaction between thiobarbituric acid (TBA) with MDA, a secondary product of lipid peroxidation (LPO) formation at $\mathrm{pH} 4$. Colorimetrically, OD value was recorded at $532 \mathrm{~nm}$ which indicates the extent of peroxidation. The activity of LPO was expressed as $\eta \mathrm{mol} / \mathrm{mg}$ protein.

Superoxide dismutase (SOD): SOD was estimated as per the method described by Madesh and Balasubramanian [18]. It involves the generation of superoxide by pyragallol autoxidation and the 
Table-1. Frequency of bone marrow micronuclei, LPO, SOD and glucose levels in control, diabetic and aqueous extract treated diabetic groups on day 91 of the experiment.

\begin{tabular}{lccc}
\hline Parameter & Control (Group - I) & Diabetic control (Group - II) & B lack Cumin (Group - III) \\
\hline$\% \mathrm{MN}$ in PCEs & $0.43 \pm 0.2^{\mathrm{c}}$ & $1.54 \pm 0.12^{\mathrm{a}}$ & $0.96 \pm 0.17^{\mathrm{b}}$ \\
$\% \mathrm{MN}$ in NCEs & $0.40 \pm 0.2^{\mathrm{c}}$ & $1.33 \pm 0.11^{\mathrm{a}}$ & $0.89 \pm 0.11^{\mathrm{b}}$ \\
P/N ratio & $1.44 \pm 0.07^{\mathrm{a}}$ & $1.04 \pm 0.07^{\mathrm{a}}$ & $1.23 \pm 0.29^{\mathrm{a}}$ \\
SOD & $13.1 \pm 0.60^{\mathrm{a}}$ & $4.4 \pm 0.13^{\mathrm{c}}$ & $8.5 \pm 0.40^{\mathrm{b}}$ \\
LPO & $4.8 \pm 0.27^{\mathrm{c}}$ & $10.1 \pm 0.35^{\mathrm{a}}$ & $6.1 \pm 0.42^{\mathrm{b}}$ \\
Glucose (mg/dl) & $115.4 \pm 3.0^{\mathrm{c}}$ & $313.6 \pm 8.5^{\mathrm{a}}$ & $166.8 \pm 4.6^{\mathrm{b}}$ \\
\hline
\end{tabular}

Means bearing different superscripts $(a, b, c)$ within the column differ significantly $(p<0.05)$

inhibition of Superoxide-dependent reduction of the tetrazolium dye MTT [3-(4-5 dimethyl thiazol 2-xl) 2, 5 diphenyl tetrazolium bromide] to its formazan, measured at $570 \mathrm{~nm}$. The reaction was terminated by the addition of dimethyl sulfoxide (DMSO), which helps to solubilize the formazan formed. Colorimetrically, OD value was taken at $570 \mathrm{~nm}$ and is expressed as SOD Units (1 unit of SOD is the amount $(\mu \mathrm{g})$ of haemoglobin required to inhibit the MTT reduction by $50 \%)$.

Blood glucose estimation: The tail vein was pricked with 24 gauge needle to collect minute amount of blood and a glucose strip was applied over the area to blot the blood and to immediately measure the fasting glucose levels (mg / dl) using the Dr. Morpen glucometer (Morepen Laboratories Ltd., New Delhi, India).

Statistical analysis: Data are expressed as Mean and standard error (SE). Analysis of variance (ANOVA) was used to find out significant variations in the different groups of studied. A P $<0.05$ was considered statistically significant.

\section{Results}

After the administration of NicotimamideStreptozotocin (NA-STZ) @ 110-45 mg/kg b.wt i.p. in wistar rats, non-insulin dependent diabetes mellitus (NIDDM) was induced. These diabetic rats showed significantly higher number of micro-nucleated cells both in normochromatic and polychromatic RBCs besides reducing the $\mathrm{P} / \mathrm{N}$ ratio as compared to the normal control animals (group- I). Administration of Nigella sativa at $500 \mathrm{mg} / \mathrm{kg}$ P.O reduced the incidences of micronuclei $(\mathrm{MN})$ in PCEs $(P<0.05)$ and NCEs $(P<$ $0.05)$ but did not alter the $\mathrm{P} / \mathrm{N}$ ratio as compared to the Diabetic (Group-II) animals.

Diabetic animals (group-II) significantly $(P<$ 0.05 ) increased the lipid peroxidation activity and reduced the levels of antioxidants enzyme SOD as compared to the control group (I). Nigella sativa at the dose $500 \mathrm{mg} / \mathrm{kg}$ orally for 90 days showed significant $(P<0.05)$ decrease in the lipid peroxidation levels and enhanced the levels of antioxidant enzyme (SOD) as compared to experimental NIDDM (groups- II) rats. In addition to these, Nigella sativa significantly $(P<0.05)$ reduced the hyperglycemia in the diabetic rats (Table- 1 ).

\section{Discussion}

In present experiment, the NIDDM was induced after the administration of Nicotimamide-Streptozo- tocin and this condition produced significant defects in RBCs in terms of creating micronuclei and enhancing the level of oxidative stress in diabetic rats (group-II). The significant finding is that the Nigella sativa treated diabetic rats (group- III) showed decreased incidences of micronuclei in erythrocytes and blood glucose along with an enhanced antioxidant activity.

The bone marrow micronuclei test was devised for evaluating the ability of test article to induce numerical and/or structural DNA damage [19]. Both kinds of damages are associated with the appearance and/or progression of tumors and with adverse reproductive and developmental outcomes. In this study, there was an increase in the frequency of micronuclei in polychromatic and normochromatic cells in group-II as compared to normal control rats (group- I). The $\mathrm{P} / \mathrm{N}$ ratio was also decreased in diabetic groups but not significantly.

High blood glucose level indicates over production of reactive oxygen species by the mitochondrial electron transport chain. High reactivity of the reactive oxygen species induces chemical changes in virtually all the cellular components leading to DNA and protein modifications and lipid peroxidation [19-20]. Further, the agent treated animals and vehicle control animals provide the cytotoxic index as the damage tend to decrease the P/N ratio [20]. In this study, an experimental type-II DM was induced by the administration of nicotinamide (NA) and STZ. The administration of NA prior to STZ partially protects the $\beta$-cells against the cytotoxic damages of STZ in adult rats [5] and the changes that follow is reported to be closer to T2DM especially with regard to the insulin response towards glucose [21]. The observations indicated that the administration of NA-STZ to the Wistar rats had increased the frequency of $\mathrm{MN}$ in the erythrocytic population and had reduced the $\mathrm{P} / \mathrm{N}$ ratio. Earlier findings suggest that the changes observed in the frequency of $\mathrm{MN}$ and $\mathrm{P} / \mathrm{N}$ ratio in the diabetic condition is mainly due to the oxidative stress mediated cytogenetic damages [19-20]. The possible mechanisms involved are: increased polyol pathway flux and advanced glycation endpoint formation, activation of protein kinase $\mathrm{C}$ (PKC) isoforms and an increased hexosamine pathway flux could all be the effects of hyperglycemia-induced overproduction of superoxide by the mitochondrial electron-transport chain [18, 22]. In addition, the diabetic condition is also reported to impair the response of the antioxidant genes to hyperglycemia, leading to reduced expression of antioxidant 
enzymes [20].

In present study, rats treated with black cumin (groups -III) decreased the elevated LPO and also increased the antioxidant enzyme activities. Schettler et al., [23] suggested that the increased level of activity of oxygen metabolites leads to reduced antioxidant defense system. It has also been suggested by Kennedy and Baynes [24] that the decreased antioxidant enzyme activity in diabetes mellitus is due to non-enzymatic glycosylation of the enzymes. So, in addition to its antidiabetic effects, black cumin may also have antioxidative properties that will be useful for therapeutic purposes. The results of the this study indicated that the protective effects of black cumin may be due to inhibition of LPO activity as a result of its antioxidant nature.

Measurement of the effect of $N$. sativa oil on gluconeogenesis and liver glucose production helps to clarify part of the hypoglycemic mechanism since hepatic glucose production through gluconeogenesis is known to contribute to hyperglycemia in diabetic patients. Research on isolated hepatic cells showed a significant decrease in glucose production from gluconeogenic elements like glycerol, alanine and lactate in Nigella sativa oil-treated animals as compared to the untreated animals [25]. This significant decrease in liver glucose output suggests that the observed antidiabetic action of $\mathrm{N}$. sativa oil is at least partially mediated through an effect on hepatic gluconeogenesis [26].

\section{Conclusion}

Rats with experimentally induced non insulin depended diabetes mellitus by the administration of NA-STZ increased the blood glucose level, germinal cell damage besides increasing the level of oxidative stress. On the other hand, treatment with Nigella sativa at $500 \mathrm{mg} / \mathrm{kg}$ had beneficial effects as shown by a decreased the blood glucose level, frequency of micronuclei formation and enhanced the antioxidant status but did not increase the $\mathrm{P} / \mathrm{N}$ ratio.

\section{Authors' contributions}

TJS carried out the present investigation under the guidance of DVJ. BJP helped in necropsy and sample collection. DVJ and CMM drafted and revised the manuscript.All authors read and approved the final manuscript.

\section{Acknowledgements}

The authors are thankful to the Dean, College of Veterinary Science and Animal Husbandry, S. D. Agricultural University for providing necessary facilities and fund to carry out the work.

\section{Competing interests}

The authors declare that they have no competing interests.

\section{References}

1. American Diabetes Association Diagnosis and classification of diabetes mellitus. (2010) J Diabetes Care. 33: S62-9.
2. Oktayoglu, G.S,, Basaraner, H., Yanardag, R., Bolkent, S. (2009) The effects of combined treatment of antioxidants on the liver injury in STZ diabetic rats. Diges Dis Sci. 54: $538-46$.

3. American Diabetes Association Diagnosis and classification of diabetes mellitus. (2010) Diabetes Care. 33: S62-10.

4. Mohan, V., Sandeep, S., Deepa, R., Shah, B. and Varghese, C. (2007) Epidemiology of type 2 diabetes: Indian scenario. Indian J. Med. Res., 125: 217-230.

5. Sharma, P., Jha, A.B., Dubey, R.S., and Pessarakli, M. (2012) Reactive Oxygen Species, Oxidative Damage, and Antioxidative Defense Mechanism in Plants under Stressful Conditions. Journal of Botany. Hindawi Publishing Corporation. Article ID 217037.

6. Blasiak, J., Arabski, M., Krupa, R., Wozniak, K., Zadrozny, M., Kasznicki, J., Zurawska, M. and Drzewoski, J. (2009) DNA damage and repair in type 2 diabetes mellitus. Mutat Res, 554: 297.

7. Masiello, P., Broca, C., Gross, R., Roye, M., Manteghetti, M. and Hillaire-Buys, D. (1998) Experimental NIDDM: Development of new model in adult rats administered streptozotocin and nicotinamide. Diabetes. 47: 224.

8. Simona, S., Yves, G., Chakradhar, V., Hanna, E.A. and Samy, L.H. (2009) Mechanism of oxidative DNA damage in diabetes: tuberin inactivation and down regulation of DNA repair enzyme 8-oxo-7, 8-dihydro-20-deoxyguanosineDNA glycosylase. Diabetes. 57: 2626-2636.

9. Devi, K., Rabbani, S. I., and Khanam, S. (2009). Inhibitory effect of Glimepiride on nicotinamide-streptozotocin induced nuclear damages and sperm abnormality in diabetic Wistar rats. Indian J. Exp. Biol. 47 (10): 804-810.

10. Jenssen, C. and Ramel, C. (1980) The micronucleus test as a part of a short-term mutagenicity test program for the prediction of carcinogenicity evaluated by 143 agents test. Mutat. Res. 75: 191-202.

11. Chauhan, L. K. S., Pant, N., Gupta, S. K. and Srivastava, S. P. (2000) Induction of chromosome aberrations, micronucleus formation and sperm abnormalities in mouse following carbafuran exposure. Mutat Res. 465: 123.

12. Rabbani, S.I., Devi, K., Khanam, S., (2009) Inhibitory effect of glimepiride on the nicotinamide-streptozotocin induced nuclear damages and sperm abnormalities in diabetic Wistar rats. Indian J. Exp. Biol. 47 (10): 804-810.

13. Gazioano, T. A., Galea, G. and Reddy, K. S. (2007) Scaling up interventions for chronic disease prevention: the evidence. Lancet. 370 (9603): 1939-1946.

14. Kanter, M. (2008) Effects of Nigella sativa and its major constituent, thymoquinone on sciatic nerves in experimental diabetic neuropathy. Neurochem Res. 33(1):87-96.

15. Kasim, S. H., Al-Mayah, Nada M. Al-Bashir and Bader M. Al-Azzaw. (2012) In Vivo Efficacy of Nigella Sativa Aqueous Seed Extract Against Metacestode of Echinococcus Granulosus. Med J Babylon.9(1): 140-151.

16. MacGregor, J. T., Heddle, J. A., Hite, M., Margolin, B. H, Ramel, C., Salamone, M.F., Tice, R.R. and Wild, D. (1987) Guidelines for the conduct of micronucleus assays in mammalian bone marrow erythrocytes. Mutat Res. 189 (2): $103-12$.

17. Shafiq-Ur-Rehman. (1984) Lead-induced regional lipid peroxidation in brain. Toxicol. Letters. 21:333-337.

18. Madesh, M and Balasubramanian, K. A. (1998) Microtiter plate assay for superoxide dismutase using MTT reduction by superoxide. Indian J. Biochem. Biophys. 35 (3):184-188.

19. Piconi, L., Quagliaro, L. and Ceriello, A. (2003) Oxidative stress in diabetes. Clin. Chem. Lab. Med. 41:1144-49.

20. Valko, M., Leibfritz, D., Moncol, J., Cronin, M. T., Mazur, M. and Telser, J. (2007) Free radicals and antioxidants in normal physiological functions and human disease. The Int $J$ Biochem. Cell Biol. 39:44-84.

21. Yasui, Y., Kim, M. and Tanaka, T. (2008). PPAR ligands for cancer chemoprevention. PPAR Research. Article ID: 548919. 1-10. 
22. Garcia-Ramirez, M., Francisco, G., Garcia-Arumi, F., Hernandez, C., Martinez, R., Andreu, A. L., et al. (2008) Mitochondrial DNA oxidation and manganese superoxide dismutase activity in peripheral blood mononuclear cells from type-2 diabetic patients. Diabetes Metab. 34:117-24.

23. Schettler, V., Wieland E., Verwiebe, R., Schuff-Werner, P., Scheler, F. and Oellerich, M. (1994) Plasma lipids are not oxidized during hemodialysis. Nephron. 67:42-47.

24. Baynes, J.W. (1991) Role of oxidative stress in development of complications in diabetes. Diabetes. 40:405-412.

25. Ishikawa, Y., Watanabe, K., Takeno, H. and Tani, T. (1998)

Effect of the novel oral antidiabetic agent HQL-975 on oral glucose and lipid metabolism in diabetic $\mathrm{db} / \mathrm{db}$ mice. Arzneim Forsch/Drug Res. 48: 245-250.

26. Al-Awadi, F. M., Fatania, H.and Shamte, U. (1991) The effect of a plant mixture extract on liver gluconeogenesis in streptozotocin induced diabetic rats. Diabetes Res. 18: 163168.

$* * * * * * * *$ 